\title{
Caracterização Da Prescrição Médica Em Um Hospital Universitário Do Centro Do Estado Do Rio Grande Do Sul
}

\author{
Santos, M.M.; Pontel, R.C.; Hodali, N.F.; Flores, L.M.; Campos, M.M.A.; \\ Apresentador: Regis Carpes Pontel \\ Premiados - Menção Honrosa: Destaque acadêmico
}

\section{Resumo}

Introdução: A prescrição, primeira etapa do ciclo de utilização de medicamentos, constitui-se em importante ato na conduta diária dos hospitais. Este estudo objetiva caracterizar a prescrição médica em um hospital universitário. MÉTODO: Estudo de delineamento transversal, envolvendo pacientes do $3^{\circ}$ e $5^{\circ}$ andar de um hospital universitário do centro do Rio Grande do Sul. Os dados da prescrição foram avaliados e caracterizados a partir de um escore para acompanhamento farmacêutico. O escore identifica o paciente em termos de risco (alto, moderado ou baixo) de acordo com características da prescrição: quantidade de medicamentos uso de medicamentos endovenosos (MEV), medicamentos potencialmente perigosos (MPP), utilização de suporte nutricional, idade, presença de problemas renais/hepáticos (PRH), problemas cardíacos/pulmonares (PCP) e imunossupressão/ imunodepressão (IMS/D). A análise dos dados foi realizada com o auxílio do Software EpiInfo®. RESULTADOS: Foram analisadas 176 prescrições (de pacientes atendidos por 15 especialidades médicas), no período de jul/2013 a dez/2013. Destes, a maioria era não idoso 105 (60\%) e não apresentava PRH 141 (80\%), PCP 101 (57\%) e/ou IMS/D 149 (85\%). Do total, 53 (30\%) usavam 16 ou mais medicamentos, 131 (74\%) tinham 4 ou mais MEV prescritos e 133 (76\%) usavam 4 ou mais MPP, perfazendo $116(66 \%)$ pacientes de alto risco (AR). Interessante notar que dentre os pacientes de AR, 59 (51\%) são idosos, ou seja, há uma divisão igualitária do risco independente da idade. Entretanto, dentre os idosos, 59 (83\%) foram considerados $\mathrm{AR}, \mathrm{RP}=2,7(1,5-4,7), \mathrm{p}<0,001$. A maioria dos pacientes com PCP 59 (82\%) faziam uso de 16 ou mais medicamentos, $\mathrm{RP}=1,9(1,1-3,4), \mathrm{p}<0,05$. Dos pacientes AR, 107 (92\%) utilizavam 11 ou mais medicamentos, 99 (85\%) utilizava MPP, 103 (89\%) utilizava 4 ou mais MEV e $35(19,9 \%)$ pertenciam à especialidade de cirurgia geral. CONCLUSÕES: A metodologia utilizada é importante, pois permite aos médicos vislumbrar os pacientes com maior risco de desenvolver problemas relacionados ao uso de medicamentos, como interações medicamentosas, incompatibilidades farmacêuticas e de horários, dose incorreta, duplicidade terapêutica e via de administração incorreta. Considerando o grande volume de prescrições nos hospitais, o reduzido número de farmacêuticos hospitalares e a carência de serviços de informação sobre medicamentos, este estudo sobre a prescrição torna-se bastante relevante no contexto em que está inserido.

\section{Referência:}

Santos, M.M.; Pontel, R.C.; Hodali, N.F.; Flores, L.M.; Campos, M.M.A.;. Caracterização Da Prescrição Médica Em Um Hospital Universitário Do Centro Do Estado Do Rio Grande Do Sul. In: II Congresso Brasileiro de Medicina Hospitalar - II CBMH [= Blucher Medical Proceedings, vol.1, num.5] São Paulo: Editora Blucher, 2014. p.6

DOI 10.5151/medpro-II-cbmh-040 